\title{
Adalimumab treatment in Crohn's disease: an overview of long-term efficacy and safety in light of the EXTEND trial
}

This article was published in the following Dove Press journal:

Clinical and Experimental Gastroenterology

29 August 2013

Number of times this article has been viewed

\author{
Amon Asgharpour \\ Jianfeng Cheng \\ Stephen J Bickston \\ VCUHS Center for Inflammatory \\ Bowel Disease, Virginia \\ Commonwealth University Health \\ Center, Richmond,VA, USA
}

Correspondence: Stephen J Bickston VCUHS Center for Inflammatory

Bowel Disease, Virginia Commonwealth University Health Center, Box 98034I,

Richmond, VA 23298-034I, USA

$\mathrm{Tel}+\mathrm{I} 8048289159$

Fax +l 8048284945

Email sbickston@mcrh-vcu.edu

\begin{abstract}
The advent of anti-tumor necrosis factor (TNF) therapies revolutionized the treatment of inflammatory bowel disease. Adalimumab is a subcutaneous anti-TNF agent indicated for use in patients with moderate-to-severe Crohn's disease and those with moderate-to-severe ulcerative colitis. In both diseases, it can be used for both induction of remission and for maintenance of remission. This review focuses on its use in Crohn's disease as described in the EXTEND (Extend the Safety and Efficacy of Adalimumab through Endoscopic Healing) trial. Several clinical trials using traditional instruments to measure clinical response have had endoscopic substudies looking for endoscopic healing. The EXTEND trial is the first to use mucosal healing on endoscopy as a primary endpoint for patients with moderate-to-severe Crohn's disease and baseline ulcerative disease treated with continuous adalimumab. In this well designed trial, the primary endpoint was narrowly missed, but the secondary endpoints further the notion that mucosal healing should be a more mainstream measure of drug efficacy. How this will translate from clinical trials to the clinic is not yet clear, but identifying noninvasive markers for mucosal healing, and understanding the implications of mucosal healing for safety, resource utilization, and quality of life are all worthy targets for further study. The aim of this review is to understand the role of mucosal healing, safety profile, and efficacy in patients treated with anti-TNF therapy, with particular attention to adalimumab and the EXTEND trial.
\end{abstract}

Keywords: Crohn's disease, tumor necrosis factor, adalimumab, clinical trials, inflammatory bowel disease

\section{Introduction}

Crohn's disease is a chronic, immune-mediated gastrointestinal disorder that can involve any portion of the gastrointestinal tract from the mouth to the anus, but primarily involves the terminal ileum and the colon. The natural history of Crohn's disease is characterized by recurring episodes of inflammation and ulcerations in the gastrointestinal tract, leading to complications that necessitate hospitalization, surgery, and adjustment in medical therapies. ${ }^{1,2}$ Clinical response and remission have primarily been measured by standardized indices such as the Crohn's Disease Activity Index (CDAI) as targets. ${ }^{3}$ Such indices are rightfully criticized for many shortcomings, but clinical experience and landmark cooperative studies showed a disconnect between clinical response and radiologic improvement. ${ }^{4}$ This spawned some learned helplessness regarding tangible mucosal changes as clinical endpoints. This was countered by a European collaboration to develop a valid instrument for scoring endoscopic findings in Crohn's disease, called the Crohn's Disease Endoscopic Index of Severity (CDEIS). ${ }^{5}$ Patient acceptance and the costs associated with using endoscopic tests in clinical trials 
have been an obstacle to using the CDEIS in large Phase III studies, but several modern trials have had smaller and nested substudies using endoscopic scoring. ${ }^{6-9}$ There is a growing body of evidence suggesting that patients with active mucosal inflammation who achieve mucosal healing have prolonged clinical remission, longer time to relapse, and a reduction in the need for hospital admissions and surgeries..$^{6,10-12}$ These data suggest that clinical trials should pay more attention to mucosal healing and a reduction in the need for hospitalizations and surgery as endpoints. ${ }^{13-15}$

With the advent of anti-tumor necrosis factor (TNF) $\alpha$ compounds, there have been new options for treatment of patients with Crohn's disease who are refractory or intolerant to conventional therapies (aminosalicylates, antibiotics, corticosteroids, and immunomodulatory agents), and with fistulizing disease. ${ }^{16,17}$ Infliximab (Remicade ${ }^{\circledR}$, Janssen Biotech Inc, Malvern, PA, USA) is the most studied drug in this class and is an intravenously administered recombinant chimeric monoclonal IgG antibody. Adalimumab (Humira ${ }^{\circledR}$, Abbott Laboratories, Abbott Park, IL, USA) is a subcutaneously administered fully human IgG monoclonal antibody and carries the advantages of self-administration and potentially decreased immunogenicity. ${ }^{18}$ Certolizumab pegol (Cimzia ${ }^{\circledR}$, UCB, Atlanta, GA, USA) is a subcutaneously administered PEGylated anti-TNF $\alpha$ humanized antigen-binding fragment (Fab') of a monoclonal antibody that has been conjugated to polyethylene glycol. ${ }^{19}$

The EXTEND (Extend the Safety and Efficacy of Adalimumab through Endoscopic Healing) trial was the first trial with adalimumab to use mucosal healing as its primary endpoint. EXTEND was a randomized, doubleblind, placebo-controlled, maintenance/withdrawal trial of adalimumab. Participants had moderate-to-severe ileocolonic Crohn's disease with evidence of mucosal ulceration at baseline endoscopy. They received open-label induction therapy with adalimumab. ${ }^{20}$ This paper reviews the impact of the EXTEND trial by Rutgeerts et al on long-term treatment of Crohn's disease patients with adalimumab. ${ }^{20}$

\section{Mechanism of action}

Tumor necrosis factor is a cytokine that mediates many biologic processes, one of which is that it initiates and perpetuates a defense response to local injury. ${ }^{21} \mathrm{TNF}$ plays an important role in the regulation of a cascade of pathogenic events marked by a rapid induction of cytokines and acutephase proteins such as interleukin- 6 and C-reactive protein. ${ }^{22}$ It has been demonstrated that patients with Crohn's disease have higher concentrations of TNF in their intestinal mucosa compared with healthy controls. ${ }^{23}$ The chronic inflammation associated with Crohn's disease is related to a sustained immune response. It has yet to be determined whether this is an appropriate response to an unknown pathogen or an inappropriate response to normal gut contents. The evidence suggests that there is a lack of apoptosis in the inflamed tissue of patients with Crohn's disease. ${ }^{24}$ Anti-TNF agents used in patients with Crohn's disease have been shown to increase the apoptosis level of T cells in the lamina propria and blood monocytes. ${ }^{25}$

Following anti-TNF therapy, reductions of the chemokines known as macrophage chemoattractant protein-1, macrophage inflammatory protein-1 alpha, and RANTES (regulated on activation, normal T-cell expressed and secreted) are detected by immunohistochemistry in the gut mucosa. ${ }^{23}$ These TNF-regulated chemokines are theorized to play a critical role in recruiting macrophages and $\mathrm{T}$ cells to form granulomas, a hallmark of inflammation in Crohn's disease. ${ }^{22}$ Thus, anti-TNF agents play a role in immune suppression by affecting the downstream effects of TNF on cytokines, chemokines, acute phase reactants, apoptosis, and inflammation.

\section{Treatment candidates}

Patients chosen for the EXTEND trial carried a diagnosis of ileocolonic Crohn's disease for at least 4 months and had moderate-to-severe ileocolonic Crohn's disease with a CDAI score of 220-450 points. Subjects that had documented mucosal ulceration on ileocolonoscopy during study screening were selected for inclusion.

Patients could receive concomitant therapy with azathioprine, 6-mercaptopurine, and methotrexate if they had been receiving these medications for at least 12 weeks and at a stable dose for at least 4 weeks prior to baseline assessment. Concomitant therapy with Crohn's diseaserelated antibiotics and aminosalicylates was also allowed if given at stable doses at least 4 weeks prior to baseline. Patients who had received anti-TNF agents in the past were permitted to continue unless they received adalimumab in the past or had been deemed a primary nonresponder. Steroid treatment with prednisone and budesonide was allowed if given at stable doses for at least 2 weeks prior to baseline.

Patients who had received anti-TNF agents within 8 weeks of baseline assessment were excluded from the EXTEND trial. Patients who had previously received adalimumab or 
natalizumab, a monoclonal antibody to alpha-4 integrin, were excluded.

\section{Study design}

All eligible patients in the EXTEND trial received open-label induction therapy with adalimumab $160 \mathrm{mg}$ at week 0 and $80 \mathrm{mg}$ at week 2 . The patients were then assessed at week 4 with the CDAI and randomized to receive maintenance adalimumab at a dose of $40 \mathrm{mg}$ every other week or placebo. Clinical response was defined by a decrease in CDAI of at least 70 points from baseline (CR-70). Patients who did not achieve CR-70 or had flares on two consecutive visits 2 weeks apart at or after 8 weeks into the study could receive open-label maintenance adalimumab $40 \mathrm{mg}$ every other week for the duration of the study. If patients continued to have flares or nonresponse, they could have dose escalation of adalimumab to $40 \mathrm{mg}$ weekly. Investigators were allowed to discontinue treatment with adalimumab if the patient continued to experience nonresponse at an escalated dose. All patients received endoscopy at baseline, week 12, week 52, and at the time of switch to open-label adalimumab if this was done. ${ }^{20}$ The EXTEND trial enlisted 135 eligible patients to participate in the study, of whom 129 were randomized at week 4 to placebo or adalimumab.

\section{Endpoints}

The primary endpoint of the EXTEND study was mucosal healing, lack of mucosal ulceration at week 12 on ileocolonoscopy. Other endpoints included mucosal healing at week 52 on endoscopy, CDEIS remission at weeks 12 and 52, CDAI remission defined as a decrease of at least 100 points from baseline (CD-100), and CR-70 at weeks 12 and $52 .^{20}$

The CDAI has been used in numerous trials in the treatment of Crohn's disease and is based on some objective clinical components, such as weight loss and anemia, but the score is mainly comprised of subjective clinical information obtained from patients regarding stool frequency, degree of abdominal pain, and overall sense of well-being. While reductions in the scores are often used as a means of determining clinical response, it does not provide information on mucosal inflammation and, due to its subjective nature, has been cited as a culprit for high placebo responses in clinical trials. ${ }^{26,27}$ The subjectivity of the CDAI can produce a disconnect between the clinical and endoscopic findings. The advantage of this scoring system is the noninvasive nature of determining disease activity. Scores of 150 and less are associated with quiescent disease and scores greater than 450 are associated with extremely severe disease. ${ }^{26}$ Most clinical trials have used a reduction of the CDAI by at least 70 points from baseline as an endpoint. ${ }^{8,28} \mathrm{~A}$ delta of 100 points has been suggested as more robust. ${ }^{29}$

Currently, there is no standard validated definition for mucosal healing in the literature on inflammatory bowel disease, making comparison between studies difficult, but scoring systems using gross endoscopic findings have been implemented as a means of comparing patients. ${ }^{30,31}$ The CDEIS is the gold standard for mucosal healing in Crohn's disease and its advantages are that it is validated and reproducible. ${ }^{4}$ The CDEIS uses variables such as superficial and deep ulceration, ulcerated and nonulcerated stenosis, and surface area of ulcerated and diseased segments to obtain an index score. The CDEIS does require training to use accurately and can prove cumbersome if used in clinical trials. There are other simplified scoring systems for mucosal healing, such as the Simple Endoscopic Score for Crohn's Disease, which is a simplified version of the CDEIS, ${ }^{32}$ the Mayo Endoscopic score, ${ }^{27,33}$ and the Rutgeerts score, ${ }^{34}$ all of which are useful tools for determining the degree of endoscopic healing and to compare levels of healing between different groups of patients. While these other scoring systems are useful and easier to obtain, the CDEIS remains a higher standard for determining the severity of mucosal disease in patients with Crohn's disease. These scoring systems all have limitations and there exist variations in operator interpretation of lesions with overestimations of subtle lesions that are large and underestimations of short lesions.

Another issue that arises when discussing mucosal healing is the role of histologic/microscopic healing obtained from biopsies. ${ }^{35}$ Absence of any microscopic inflammation has been touted as the ultimate goal in treating patients with Crohn's disease. Just as there is no universally embraced definition of mucosal healing, there is also no absolute standard for histologic healing. It has been demonstrated that patients may have achieved gross endoscopic healing on ileocolonoscopy that is not paralleled in histology. ${ }^{36}$ Furthermore, while a standardized histologic scoring system exists for both ulcerative colitis and Crohn's disease, ${ }^{37}$ there is variation in reported scoring secondary to the interpretation of pathologists. The surgical literature suggests that the presence of microscopic disease at resection margins does not change clinical outcomes, making the case for conservative resections to eliminate only gross disease. ${ }^{38}$ While histologic regression of disease is important, using it as an endpoint 
makes clinical trials more costly and cumbersome, and this has been discouraged. ${ }^{39}$

\section{Safety}

The most worrisome safety concern that arises for patients receiving adalimumab is the potential risk of serious infections, including tuberculosis and malignancy, because these complications have been reported with other TNF antagonists. ${ }^{40,41}$ Patients should be screened for tuberculosis infections with a negative purified protein derivative test and chest radiography because reactivation of tuberculosis has been reported. ${ }^{40}$ The increased risk of infection stems from a common desired mechanism of treatment, ie, suppression of the immune system. ${ }^{22}$ Other reported complications with this class of drug include congestive heart failure, lupus-like syndrome, and demyelinating disorders. ${ }^{42-44}$

In the EXTEND trial, there were no reported deaths, cases of malignancy, congestive heart failure, demyelinating disorder, allergic reaction, lupus-like syndrome, or infections with tuberculosis. ${ }^{20}$ Infections were reported in both placebo (34\%) and maintenance adalimumab groups (55\%), with a statistically significant increase in the number of infections in the adalimumab maintenance group compared with placebo. When these incidences were normalized for patientyears of exposure, there was no statistically significant difference between these groups. ${ }^{20}$ Serious infections seen in the EXTEND trial were abscesses, herpes zoster, and tonsillitis.

The safety profile in the EXTEND trial is similar to that seen in patients receiving TNF antagonists in other studies. A large-scale meta-analysis looking at the long-term safety profile of adalimumab across different diseases including rheumatoid arthritis, ankylosing spondylitis, psoriatic arthritis, and Crohn's disease in more than 23,000 patients over 12 years showed serious infection as the most common serious adverse event, with no new safety concerns revealed. ${ }^{45}$ Another meta-analysis including 13 randomized controlled trials with over 4,000 patients looking at serious adverse events of anti-TNF- $\alpha$ treatment in patients with Crohn's disease showed that TNF antagonists, especially adalimumab, could actually decrease the incidence of serious adverse events without an increased risk of malignancy or serious infections. ${ }^{46}$

\section{Efficacy}

Several studies prior to the EXTEND trial, using clinical response and remission as endpoints, have established the efficacy of adalimumab. The CLASSIC-I (Clinical
Assessment of Adalimumab Safety and Efficacy Studied as Induction Therapy in Crohn's Disease) trial, reported by Hanauer et al, demonstrated that adalimumab was more effective than placebo for induction of remission in patients with moderate-to-severe Crohn's disease naïve to anti-TNF therapy at an optimal dose of $160 \mathrm{mg}$ at week 0 followed by $80 \mathrm{mg}$ at week 2. The CLASSIC-I trial used a CDAI of less than 150 as a definition of remission. ${ }^{47}$ The GAIN (Gauging Adalimumab effectiveness in Infliximab Nonresponders) trial showed that patients who were nonresponders to infliximab could achieve clinical remission, defined as a decrease in CDAI of at least 70 points, when treated with adalimumab compared with placebo. ${ }^{48}$ Colombel et al demonstrated that patients receiving a maintenance dose of adalimumab $40 \mathrm{mg}$ every other week or weekly for up to one year achieved significantly greater remission rates than placebo at weeks 26 and 52. This trial, called CHARM (Crohn's Trial of the Fully Human Antibody Adalimumab for Remission Maintenance), used a decrease in the CDAI of at least 70 points as a definition of clinical remission. ${ }^{49}$ An extension of the CLASSIC-I trial, CLASSICII, was able to demonstrate maintenance of clinical remission, defined as a CDAI of less than 150 at week 56, in patients who received adalimumab $40 \mathrm{mg}$ weekly or every other week compared with controls. ${ }^{50}$

In regards to clinical remission, the EXTEND trial showed no statistically significant difference in CD-100 or CR-70 at week 12 between the induction only/placebo group versus the continuous adalimumab group; however, there was a statistically significant difference in CDAI remission rates in favor of continuous adalimumab compared with induction only/placebo at week 12 . In contrast, at week 52, there was a significant difference $(P=0.004)$ in CD-100 between the continuous adalimumab group (36\%) and the induction only/ placebo group (14\%). The CD-70 showed a similar difference at week 52 , with $14 \%$ of patients achieving CD-70 in the induction only/placebo group and $41 \%$ in the continuous adalimumab group. ${ }^{20}$

The most interesting results in the EXTEND trial are those related to mucosal healing. At week 12, mucosal healing was achieved in $27 \%$ of patients receiving continuous adalimumab compared with $13 \%$ for the induction-only/ placebo group $(P=0.056)$. When patients who had major protocol deviations were removed from these groups, there were significantly greater rates of mucosal healing in the continuous adalimumab group at week $12(P=0.046)$. There was a statistically significant difference in CDEIS remission, defined as a CDEIS score of $\leq 4$, favoring continuous adalimumab therapy over induction only/placebo at 12 weeks. 
There was no statistically significant difference in CDEIS responders, defined as a reduction in CDEIS of $>75 \%$ from baseline, between the two groups at week 12. ${ }^{20}$

The efficacy of maintenance adalimumab was demonstrated at week 52 , with $24 \%$ of patients receiving continuous adalimumab achieving mucosal healing and no patients with mucosal healing in the induction only/placebo group at week $52(P<0.001) .{ }^{20}$ There was also a statistically significant difference favoring continuous adalimumab therapy over induction only/placebo in regards to CDEIS remission rates and CDEIS 75\% responders. ${ }^{20}$

Subgroup analysis revealed that patients with a CDEIS of $\leq 9$ at baseline achieved significantly higher levels of mucosal healing on continuous adalimumab compared with induction only/placebo at week 12 . There was no difference in mucosal healing at week 12 in either group if the CDEIS was $>9$. At week 52, there was a significantly higher level of mucosal healing in the continuous adalimumab group compared with mucosal healing in the induction only/placebo group, regardless of CDEIS score. ${ }^{20}$

There were significantly more patients in the EXTEND trial who were receiving baseline corticosteroid therapy in the induction only/placebo arm as compared with the adalimumab maintenance group. Mucosal healing in the induction only/placebo group at 12 weeks was similar in patients with and without corticosteroid treatment at baseline. Patients receiving continuous adalimumab had similar rates of mucosal healing whether they had or had not received corticosteroid treatment at baseline. ${ }^{20}$ Corticosteroids did not seem to be a significant factor in achieving mucosal healing at week 12 in the EXTEND trial, and a lack of a mucosal healing effect with corticosteroids has been demonstrated in other studies. ${ }^{51}$

Further subgroup analysis in the EXTEND trial revealed that patients with C-reactive protein levels $\geq 1 \mathrm{mg} / \mathrm{dL}$ at baseline had significantly greater levels of mucosal healing in the continuous adalimumab group compared with the induction only/placebo group at week 12, and no difference in mucosal healing if C-reactive protein levels were $<1 \mathrm{mg}$ / $\mathrm{dL} .{ }^{20}$ In patients not receiving concomitant immuosuppressives, significantly greater mucosal healing at week 12 was seen in patients receiving continuous adalimumab than in those in the induction only/placebo treatment arm. ${ }^{20}$

\section{Pharmacoeconomics}

While TNF blockers have shown significant benefit for patients with moderate-to-severe Crohn's disease, they are a more expensive class of drugs compared with traditional therapies. Within the class of drugs (infliximab, adalimumab, and certolizumab pegol) there exist intrinsic differences. One of the major advantages of adalimumab and certolizumab over infliximab is the route of administration. Subcutaneous administration is more convenient for patients, decreases hours missed from work to receive infusions, and decreases the need for skilled professionals to administer therapy. A recent study revealed that for patients with moderate-tosevere Crohn's disease, the 12-month cost per responder was similar for adalimumab and infliximab. ${ }^{52}$ While 12 -month continuous treatment costs with TNF inhibitors are high, other studies have shown the cost benefit of sustained remission. ${ }^{53,54}$ In addition, it has been demonstrated, in a metaanalysis study, that adalimumab provides improvement in work productivity in patients with Crohn's disease. ${ }^{55}$

\section{Conclusion}

Mucosal healing on endoscopy is useful in determining response and guiding management in patients being treated for Crohn's disease. While several treatments provide clinical response and remission in Crohn's disease, some (sulfasalazine, prednisone) have fallen short in achieving mucosal healing. The only classes of drugs that have demonstrated mucosal healing in patients with Crohn's disease are purine analogs and anti-TNF agents. ${ }^{56}$ Mucosal healing is clinically relevant in that patients with active mucosal inflammation who achieve such healing have prolonged clinical remission, longer time to relapse, and a reduction in the need for hospital admissions and surgery. ${ }^{9-12}$

In clinical practice, all patients being treated for Crohn's disease should not necessarily receive endoscopy to determine mucosal healing because frequent endoscopy is costly, uncomfortable for patients, and carries inherent risks, such as perforation, bleeding, and infection, as well as the cardiopulmonary risks involved with sedation. Clinically, endoscopy should be used as any other test, ie, the information obtained should guide management. If a patient has clinically improved on therapy, and there is no clinical basis for altering treatment, endoscopy is of little use. It has been proposed, for clinical practice, that endoscopy to assess for mucosal healing be limited to patients on maximal therapy with continued symptoms and in patients where discontinuation of therapy is being considered. ${ }^{56}$

Rutgeerts et al should be applauded for their work in the EXTEND trial. This is the first study designed a priori to use mucosal healing as a primary endpoint in assessing patients with Crohn's disease treated with dalimumab. The patients allowed into this trial with moderate-to-severe ileocolonic 
Crohn's disease were from a more clinically relevant pool, in that they already had failure with prior therapies, than other trials. Patients were allowed to participate if they had failed prior anti-TNF therapy and/or immunomodulators, and were allowed to remain on corticosteroids if they were receiving them at baseline. These types of patients represent a subclass of difficult-to-treat patients we see in clinical practice and provide information beyond what has been learned in populations that are naïve to anti-TNF agents and have had shorter disease durations. $^{7}$

The week 12 endpoint of mucosal healing did not show a statistically significant difference between the continuous adalimumab group and the induction only/placebo group. This may demonstrate the effects of the induction therapy itself. ${ }^{20}$ A study published after EXTEND also showed mucosal healing at week 12 after adalimumab induction therapy. ${ }^{57}$ Mucosal healing, defined by CDEIS score, has been demonstrated as early as week 4 in a study with infliximab ${ }^{5}$ and as early as 10 weeks with certolizumab. ${ }^{58}$ That said, the efficacy of continuous adalimumab therapy was demonstrated at week 52 in the EXTEND trial and this supports the use of induction and long-term maintenance therapy with adalimumab. More recently, endoscopic remission at 3 months in patients treated with continuous adalimumab or infliximab was revealed to be a strong predictor of endoscopic healing at one year. ${ }^{59}$ Hence, there may be a role for endoscopy at twelve weeks for patients after receiving adalimumab. This could help determine treatment response; if mucosal healing is not seen, it may trigger the substitution or addition of a different medication.

A more recent post hoc analysis of the CHARM and EXTEND trials showed that patients with moderate Crohn's disease, defined as a CDAI score $\leq 300$, had the highest clinical remission rates compared with other patients in these trials at 4 weeks, with the highest levels of efficacy in patients with moderate Crohn's disease and a C-reactive protein $>1 \mathrm{mg} / \mathrm{dL} .{ }^{64}$ Patients with moderate Crohn's disease and high C-reactive protein receiving placebo had disease activity and number of hospitalizations similar to patients with severe Crohn's disease receiving placebo. ${ }^{60}$ This study suggests that there may be a role for obtaining C-reactive protein levels in patients with moderate Crohn's disease because this patient population has the highest benefit from treatment with adalimumab and are at great risk for disease progression. ${ }^{60}$

The safety profile of adalimumab in the EXTEND study was similar to that in other trials and showed no new safety concerns. The main concerns continue to be the risk of serious infections and the need for tuberculosis screening.
The risk of serious infections seems to be less concerning with further analysis, ${ }^{20,46}$ especially considering the benefits of continuous adalimumab treatment. However, because these studies do not have follow-up data beyond a year, further long-term studies are needed to confirm the safety profile of adalimumab in the treatment of Crohn's disease.

Further studies are needed to understand the efficacy and safety profile of adalimumab beyond a year of treatment. Furthermore, endpoints other than clinical response and remission should be implemented in future studies so that more can be understood about the mucosal microenvironment, the gross appearance of the mucosa, and financial impact on patients and society.

\section{Disclosure}

SJB has received honoraria and research funds from Centocor, Amgen, and Abbott. The other authors report no conflicts of interest in this work.

\section{References}

1. Peyrin-Biroulet L, Loftus EV Jr, Colombel JF, Sandborn WJ. The natural history of adult Crohn's disease in population-based cohorts. Am J Gastroenterol. 2010;105:289-297.

2. Langholz E. Ulcerative colitis. An epidemiological study based on a regional inception cohort, with special reference to disease course and prognosis. Dan Med Bull. 1999;46:400-415.

3. Best WR, Becktel JM, Singleton JW, Kern F Jr. Development of a Crohn's disease activity index. National Cooperative Crohn's Disease Study. Gastroenterol. 1976;70(3):439-444.

4. Summers RW, Switz DM, Sessions JT Jr, et al. National Cooperative Crohn's Disease Study: results of drug treatment. Gastroenterology. 1979;77(4 Pt 2):847-869.

5. Mary JY, Modigliani R. Development and validation of an endoscopic index of the severity for Crohn's disease: a prospective multicentre study. Groupe d'Etudes Thérapeutiques des Affections Inflammatoires du Tube Digestif (GETAID). Gut. 1989;30:983-989.

6. Van Dullemen HM, van Deventer SJ, Hommes DW, et al. Treatment of Crohn's disease with anti-tumor necrosis factor chimeric monoclonal antibody (cA2). Gastroenterology. 1995;109:129-135.

7. Rutgeerts P, Diamond RH, Bala M, et al. Scheduled maintenance treatment with infliximab is superior to episodic treatment for the healing of mucosal ulceration associated with Crohn's disease. Gastrointest Endosc. 2006;63:433-442.

8. Colombel JF, Sandborn WJ, Reinisch W, et al; SONIC Study Group. Infliximab, azathioprine, or combination therapy for Crohn's disease. N Engl J Med. 2010;362:1383-1395.

9. Hanauer SB, Feagan BG, Lichtenstein GR, et al; ACCENT I Study Group Maintenance infliximab for Crohn's disease: the ACCENT I randomized trial. Lancet. 2002;359:1541-1549.

10. Baert FJ, Moortgat L, Van Assche GA, et al; Belgian Inflammatory Bowel Disease Research Group, North-Holland Gut Club. Mucosal healing predicts sustained clinical remission in patients with early-stage Crohn's disease. Gastroenterology. 2010;138:463-468.

11. D'Haens G, Noman M, Baert F, et al. Endoscopic healing after infliximab treatment for Crohn's disease provides a longer time to relapse. Gastroenterology 2002;122 Suppl 4:A-100.

12. Schnitzler F, Fidder H, Ferrante M, et al. Mucosal healing predicts long-term outcome of maintenance therapy with infliximab in Crohn's disease. Inflamm Bowel Dis. 2009;15:1295-1301. 
13. Rutgeerts P, Vermeire S, Van Assche G. Mucosal healing in inflammatory bowel disease: impossible ideal or therapeutic target? Gut. 2007;56: 453-455.

14. D'Haens GR, Fedorak R, Lémann M, et al. Endpoints for clinical trials evaluating disease modification and structural damage in adults with Crohn's disease. Inflamm Bowel Dis. 2009;15:1599-1604.

15. Taylor KM, Irving PM. Optimization of conventional therapy in patients with IBD. Nat Rev Gastroenterol Hepatol. 2011;8:646-656.

16. Blonski W, Buchner AM, Lichtenstein GR, et al. Inflammatory bowel disease therapy: current state-of-the-art. Curr Opin Gastroenterol. 2011;27:346-357.

17. Clark M, Colombel JF, Feagan BC, et al. American Gastroenterological Association Consensus development conference on the use of biologics in the treatment of inflammatory bowel disease, June 21-23, 2006 Gastroenterology. 2007;133:312-339.

18. Etchevers MJ, Orda I, Richart E, et al. Optimizing the use of tumour necrosis factor inhibitors in Crohn's disease. A practical approach. Drugs. 2010;70:109-120.

19. Goel N, Stephens S. Certolizumab pegol. MAbs. 2010;2:137-147.

20. Rutgeerts P, Assche GV, Sandborn WJ, et al. Adalimumab induces and maintains mucosal healing in patients with Crohn's disease: data from the EXTEND trial. Gastroenterology. 2012;142:1102-1111.

21. Thomson AB, Gupta M, Freeman H. Use of the tumor necrosis factor-blockers for Crohn's disease. World J Gastroenterol. 2012;18: 4823-4854.

22. Tracey D, Klareskog L, Sasso EH, et al. Tumor necrosis factor antagonist mechanisms of action: a comprehensive review. Pharmacol Ther. 2008;117:244-279.

23. Van Deventer S. Tumour necrosis factor and Crohn's disease. Gut. 1997;40:443-448.

24. Sands BE. Why do anti-tumor necrosis factor antibodies work in Crohn's disease? Rev Gastroenterol Disord. 2004;4:S10-S17.

25. Di Sabatino A, Ciccocioppo R, Cinque, B, et al. Defective mucosal $T$ cell death is sustainably reverted by infliximab in a caspase dependent pathway in Crohn's disease. Gut. 2004;53:70-77.

26. Best WR, Becktel JM, Singleton JW, Kern F Jr. Development of a Crohn's disease activity index: National Cooperative Crohn's Disease Study. Gastroenterology. 1976;70:439-444.

27. Dave M, Loftus EV. Mucosal healing in inflammatory bowel disease a true paradigm of success? Gastroenterol Hepatol. 2012;8:29-38.

28. Sands B, Anderson F, Bernstein C, et al. Infliximab maintenance therapy for fistulizing Crohn's disease. N Engl J Med. 2004;350: 876-885.

29. Thia KT, Sandborn WJ, Lewis JD, et al. Defining the optimal response criteria for the Crohn's disease activity index for induction studies in patients with mildly to moderately active Crohn's disease. Am J Gastroenterol. 2008;103:3123-3131.

30. D'Haens G, Sandborn WJ, Feagan BG, et al. A review of activity indices and efficacy end points for clinical trials of medical therapy in adults with ulcerative colitis. Gastroenterology. 2007;132:763-786.

31. Sandborn WJ, Feagan BG, Hanauer SB, et al. A review of activity indices and efficacy endpoints for clinical trials of medical therapy in adults with Crohn's disease. Gastroenterology. 2002;122: 512-530.

32. Daperno M, D'Haens G, Van Assche G, et al. Development and validation of a new, simplified endoscopic activity score for Crohn's disease: the SES-CD. Gastrointest Endosc. 2004;60:505-512.

33. Schroeder KW, Tremaine WJ, Ilstrup DM. Coated oral 5-aminosalicylic acid therapy for mildly to moderately active ulcerative colitis. A randomized study. $N$ Engl J Med. 1987;317:1625-1629.

34. Rutgeerts P, Geboes K, Vantrappen G, Beyls J, Kerremans R, Hiele M. Predictability of the postoperative course of Crohn's disease. Gastroenterology. 1990;99:956-963.

35. Villanacci V, Antonelli E, Geboes K, Casella G, Bassotti G. Histological healing in inflammatory bowel disease: a still unfulfilled promise. World J Gastroenterol. 2013;19:968-978.
36. Geboes K, Dalle I. Influence of treatment on morphological features of mucosal inflammation. Gut. 2002;50 Suppl 3:III37-III42.

37. Geboes K, Riddell R, Ost A, Jensfelt B, Persson T, Löfberg R. A reproducible grading scale for histological assessment of inflammation in ulcerative colitis. Gut. 2000;47:404-409.

38. Fazio VW, Marchetti F, Church M, et al. Effect of resection margins on the recurrence of Crohn's disease in the small bowel. A randomized controlled trial. Ann Surg. 1996;224:563-571.

39. Kornbluth A, Sachar DB. Ulcerative colitis practice guidelines in adults (update): American College of Gastroenterology, Practice Parameters Committee. Am J Gastroenterol. 2004;99:1371-1385.

40. Keane J, Gershon S, Wise RP, et al. Tuberculosis associated with infliximab, a tumor necrosis factor alpha-neutralizing agent. $N$ Engl J Med. 2001;345:1098-1104.

41. Wolfe F, Michaud K. Biologic treatment of rheumatoid arthritis and the risk of malignancy. Arthritis Rheum. 2007;56:2886-2895.

42. Wolfe F, Michaud K. Heart failure in rheumatoid arthritis: rates, predictors, and the effect of anti tumor necrosis factor therapy. Am J Med. 2004;116:305-311.

43. Burmester GR, Mease P, Dijkmans BAC, et al. Adalimumab safety and mortality rates from global trials of six immune mediated inflammatory diseases. Ann Rheum Dis. 2009;68:1863-1869.

44. Mohan N, Edwards ET, Cupps TR, et al. Demyelination occurring during anti-tumor necrosis factor $\alpha$ therapy for inflammatory arthritides. Arthritis Rheum. 2001;44:2862-2869.

45. Burmester GR, Panaccione R, Gordon KB, McIlraith MJ, Lacerda AP. Adalimumab: long-term safety in 23458 patients from global clinical trials in rheumatoid arthritis, juvenile idiopathic arthritis, ankylosing spondylitis, psoriatic arthritis, psoriasis and Crohn's disease. Ann Rheum Dis. 2013;72:517-524.

46. Zhang D, Xiong B, Li X, Xu T, Yu M. Meta-analysis: serious adverse events in Crohn's disease patients treated with TNF-alpha inhibitors. Hepatogastroenterology. 2013;23:60.

47. Hanauer SB, Sandborn WJ, Rutgeerts P, et al. Human anti-tumor necrosis factor monoclonal antibody (adalimumab) in Crohn's disease: the CLASSIC-I trial. Gastroenterology. 2006;130:323-333.

48. Sandborn WJ, Rutgeerts P, Enns R, et al. Adalimumab induction therapy for Crohn disease previously treated with infliximab. A randomized trial. Ann Intern Med. 2007;146:829-838.

49. Colombel JF, Sandborn WJ, Rutgeerts P, et al. Adalimumab for maintenance of clinical response and remission in patients with Crohn's disease: the CHARM trial. Gastroenterology. 2007;132:52-65.

50. Sandborn WJ, Hanauer SB, Rutgeerts P, et al. Adalimumab for maintenance treatment of Crohn's disease: results of the CLASSIC II trial. Gut. 2007;56:1232-1239.

51. Olaison B, Sjodahl R, Tagesson C. Glucocorticoid treatment in ileal Crohn's disease: relief of symptoms but not endoscopically viewed inflammation. Gut. 1990;31:325-328.

52. Liu Y, Wu EQ, Bensimon AG, et al. Cost per responder associated with biologic therapies for Crohn's disease, psoriasis, and rheumatoid arthritis. Adv Ther. 2012;29:620-634.

53. Loftus EV Jr, Johnson SJ, Yu AP, Wu EQ, Chao J, Mulani PM. Cost-effectiveness of adalimumab for the maintenance of remission in patients with Crohn's disease. Eur J Gastroenterol Hepatol. 2009;21: 1302-1309.

54. Yu AP, Cabanilla LA, Wu EQ, Mulani PM, Chao J. The costs of Crohn's disease in the United States and other Western countries: a systematic review. Curr Med Res Opin. 2007;24:319-328.

55. Binion DG, Louis E, Oldenburg B, et al. Effect of adalimumab on work productivity and indirect costs in moderate to severe Crohn's disease: a meta-analysis. Can J Gastroenterol. 2011;25:492-496.

56. Ferrante M, Van Assche G. Medical therapy and mucosal healing. Curr Drug Targets. 2012;13:1294-1299.

57. Rismo R, Olsen T, Ciu G, et al. The effect of adalimumab for induction of endoscopic healing and normalization of mucosal cytokine gene expression in Crohn's disease. Scand J Gastroenterol. 2012;47: 1200-1210. 
58. Colombel JF, Lemann M, Bouhnik Y, et al. Endoscopic mucosal improvement in patients with active Crohn's disease treated with certolizumab pegol: week 10 and 54 results of the MUSIC trial. Gastroenterology. 2010;138:S166.

59. Bjorkesten A, Nieminen U, Sipponen T. Mucosal healing at 3 months predicts long-term endoscopic remission in anti-TNF-treated luminal Crohn's disease. Scand J Gastroenterol. 2013;48:543-551.
60. Sandborn WJ, Colombel JF, Panes J, et al. Exploring the use of adalimumab for patients with moderate Crohn's disease: subanalyses from induction and maintenance trials. J Crohns Colitis. March 18, 2013. [Epub ahead of print.] Available from: http://www.ncbi.nlm.nih.gov/ pubmed/23517933. Accesssed August 15, 2013.

\section{Publish your work in this journal}

Clinical and Experimental Gastroenterology is an international, peerreviewed, open access journal, publishing all aspects of gastroenterology in the clinic and laboratory, including: Pathology, pathophysiology of gastrointestinal disease; Investigation and treatment of gastointestinal disease; Pharmacology of drugs used in the alimentary tract;
Immunology/genetics/genomics related to gastrointestinal disease. This journal is indexed on CAS. The manuscript management system is completely online and includes a very quick and fair peer-review system. Visit http://www.dovepress.com/testimonials.php to read real quotes from published authors.

Submit your manuscript here: http://www.dovepress.com/clinical-and-experimental-gastroenterology-journal 